\title{
More on A Statistical Analysis of Log-Periodic Precursors to Financial Crashes
}

\author{
James A. Feigenbaum \\ Tippie College of Business \\ Department of Economics \\ University of Iowa \\ Iowa City, IA 55242 \\ jfeigenb@blue.weeg.uiowa.edu
}

July 2001

\begin{abstract}
We respond to Sornette and Johansen's criticisms of our findings regarding log-periodic precursors to financial crashes. Included in this paper are discussions of the Sornette-Johansen theoretical paradigm, traditional methods of identifying log-periodic precursors, the behavior of the first differences of a log-periodic price series, and the distribution of drawdowns for a securities price.
\end{abstract}


In Ref. 1], we reconsidered the evidence regarding log-periodic precursors to financial crashes. We focused much of our attention on the work of Didier Sornette and Anders Johansen [2]- [4, and they have written a detailed response [5] to our paper. We now take this opportunity to rebut their criticisms.

We made four points in [四]. First, we had some comments regarding the theory proposed by Johansen et al in [- St. Second, we reviewed the traditional curve-fitting approach for identifying log-periodicity and noted some problems with this methodology. Third and most important, we tested a prediction that comes out of the model in 画 that, in addition to the price series, the first differences of the price series should also behave log-periodically within a logperiodic precursor. In examining the first differences for the S\&P 500 during the log-periodic spell leading up to the famous October $1987 \mathrm{crash}$, we found that, if we restricted attention to the years 1980-1986 (which covers the bulk of this spell but not the last year prior to the crash), the log-periodic component of the Sornette-Johansen specification was not statistically significant at the $5 \%$ level. Finally, we weighed in on Johansen and Sornette's claim [2] that large events like the 87 crash are outliers in the distribution of drawdowns, a drawdown being the cumulative drop in an index from a local maximum to the ensuing local minimum. We will address Sornette and Johansen's response to each of these four points separately.

\section{The Sornette-Johansen Paradigm}

The economic theory proposed by Sornette and Johansen (SJ) to explain the incidence of log-periodicity in securities prices is described in [4] and summarized in Section 2 of our own paper [1]. Their model assumes that log-periodicity arises from the interaction of a large group of irrational agents who reside on a network with discrete scale invariance. They also include in their model a single rational agent who is risk neutral and has rational expectations. The introduction of this agent served two purposes. It was intended to defuse the criticism that a log-periodic bubble could be exploited by rational agents to make unbounded profits. In addition, the no-arbitrage condition derived from this agent's preferences supplies a mathematical link between the price of the security and the probability of a crash induced by the irrational agents.

In their model, they consider a security which earns no dividends, so any positive price would signify a bubble in the security price $p(t)$. The price can be described in the notation of stochastic calculus as following the process

$$
\frac{d p}{p(t)}=\mu(t) d t+\varepsilon(t)-\lambda d j,
$$

where $\mu(t)$ is a time-dependent drift and $\varepsilon(t)$ is a mean-zero noise term. The binary variable $j(t)$ keeps track of the impending crash. It will equal zero before 
a crash and one afterwards, so $d j$ will vanish except at the instant of the crash, at which time the price of the security will fall by the factor $\lambda$. (A more general model would have $\lambda$ be the mean of a distribution of possible crash sizes.) The no-arbitrage condition is then a martingale condition on the price:

$$
E[d p]=0 .
$$

One observation made in [1], which was also made by Ilinski [6], is that such a no-arbitrage condition precludes the possibility of making positive profits with certainty. That is why it is called a no-arbitrage condition. At this point, it is important to note that what an economist means when using the term profit differs from what an accountant or the typical layperson would mean. Economic profit is the surplus of revenue over opportunity cost, opportunity cost being the stream of income that is lost because one did not allocate the resources in question to some other purpose. In this context, the opportunity cost will be the income lost because one did not invest one's resources in the best possible alternative manner. When we say that the no-arbitrage condition precludes the possibility of making a certain, positive profit, we do not mean that investors can earn no revenue from trading in this security. Rather, we mean that an agent cannot improve on a strategy which earns zero expected return.

In [5], SJ argue incorrectly that, in fact, positive profits could be earned by a savvy investor in their model. They make a distinction between average returns and conditional returns. The no-arbitrage condition (2) requires that the average unconditional return be zero. However, the return conditional on no crash having occurred, represented by $\mu(t)$, can and generally will be nonzero.

This is true, but they are missing the point. Investors are not interested in conditional returns. They cannot depend on a crash not happening. If they could simply ignore the possibility of a crash, there would be no bubble. So yes, it is possible that, after the fact, an investor might find he has earned some money by trading in this security. However, before the fact, he will have just as much expectation that he might lose that same amount. There is no way to construct a trading strategy that will exploit this bubble to earn an expected return greater than zero. That is the upshot of the no-arbitrage condition. If one is going to presume that the Sornette-Johansen paradigm is valid-keeping in mind we do not advocate that presumption-then it would be quite irrational to attempt to devise a money-making strategy which uses log-periodicity to forecast crashes. One might as well spend one's time seeking to invent a perpetual motion machine.

As SJ note [5], there is some empirical evidence that the efficient market hypothesis is false and that it might be possible to earn positive profits relative to a standard diversification strategy. However, this evidence does not support their model. On the contrary, it goes against the assumptions of their model.

Another point which Ilinski [6] made that we expanded upon in [1] was the criticism that SJ's model is based on the unrealistic assumption that only a single rational agent exists, who also happens to be risk neutral. In [5], SJ 
make a fair stab at loosening the risk neutrality assumption. They draw upon a stochastic discount factor (SDF) model in [7] to broaden their results and conclude that "the most general form of risk aversion does not invalidate our theory." As anyone familiar with the literature on expected utility theory should know, Cochrane's model, while certainly useful, is by no means an exhaustive model of risk averse preferences. However, this specification is presumably general enough to serve the purpose of showing their model can accomodate the possibility that their single rational agent is risk averse.

Unfortunately for SJ, the multiplicity issue will be much more difficult to tame. They note in passing [5] that "the SDF is not different from one agent to the next . . . because it describes the aggregate perception by the rational agents of the level of risks." Essentially, SJ are invoking what is known as a representative-agent model of the macroeconomy. Under certain conditions, such as complete markets, it can be shown that in equilibrium an economy of many agents can be replaced by a single imaginary agent, and, indeed, the stochastic discount factor of all the individual agents would equal the SDF for this imaginary agent [8].

The problem with this argument is that it is circular reasoning, for it requires than an equilibrium consistent with the bubble exists. They are assuming what they are supposed to be proving. One purpose of introducing the rational agent was to forestall the criticism that a rational agent might exploit a log-periodic bubble to earn unbounded profits, a situation that could not occur in equilibrium and has never been observed empirically. Quoting from Johansen et al in (4), "Lest this sound like voodoo science, let us reassure the reader that the ability to predict the critical date is perfectly consistent with the behavior of the rational agents in our model: they all know this date, the crash may happen anyway, and they are unable to make any abnormal risk-adjusted profits by using this information." Thus far, we are not reassured.

It is straightforward to construct a price process consistent with equilibrium for one rational agent, but it is considerably more difficult to accomplish this for two or more rational agents. Allowing an exotic price bubble compounds the situation further. It is more than likely that very stringent assumptions would have to be put on agents' preferences to construct a market equilibrium with a log-periodic price bubble.

Of course, one could dispense with rationality and no arbitrage, and then it is trivial to get log-periodicity in price processes as Sornette and Ide demonstrated in [9]. The trick is to combine all these elements, and so far no one has accomplished this except under highly unrealistic assumptions.

\section{Fitting the Price Curve}

The majority of [5] is devoted to attacking the third section of [1] in which we review past work on identifying log-periodicity. In their words, "We are 
obviously not going to divulge our technique and methodology for crash prediction but instead offer a few common sense guidelines to avoid the rather obvious traps in which Feigenbaum has fallen." One could just as well argue that they are the ones falling into the traps. We see only a marginal role for common sense in determining their guidelines. Their rules of thumb appear to have been chosen to produce the results they want to show. They give no econometric justification for rejecting our own inferences.

The specification that we used in [1] came from [10]. The log of the price is assumed to obey

$$
\ln (p(t))=A+B f_{1}(t)+C f_{2}(t),
$$

where

$$
f_{1}(t)=\frac{\left(t_{c}-t\right)^{\beta}}{\sqrt{1+\left(\frac{t_{c}-t}{\Delta_{t}}\right)^{2 \beta}}}
$$

and

$$
f_{2}(t)=f_{1}(t) \cos \left[\omega \ln \left(t_{c}-t\right)+\frac{\Delta_{\omega}}{2 \beta} \ln \left(1+\left(\frac{t_{c}-t}{\Delta_{t}}\right)^{2 \beta}\right)+\phi\right] .
$$

This is a specification with three linear parameters $A, B$, and $C$; and six nonlinear parameters $\omega, \beta, \Delta_{t}, \Delta_{\omega}, t_{c}$, and $\phi$. We should note that the critical time $t_{c}$ does not correspond to the time of the crash but, rather, to a time when the probability of a crash is maximized.

Econometrically, this is an extremely unfriendly model because there are so many nonlinearities. Furthermore, in the Sornette-Johansen paradigm this specification is supposed to correspond to an integral of the function $\mu(t)$ in Eq. (11), which would imply that $\ln p(t)$ is autocorrelated. As we demonstrated with a pedagogical example in Section 4 of [1], regressing an autocorrelated time series on functions of time alone can lead to spurious results, and this kind of regression is precisely what is being done when one fits a stock index to the specification (3).

The many curve fits of log-periodic spells existing in the literature are producing descriptive statistics regarding the realization of price trajectories. However, these descriptive statistics may offer no information about the structural parameters of the underlying stochastic process. SJ's primary objection [5 to our results in [1] is that we often obtain values for the nonlinear parameters which are either too large or too small relative to some unspecified standard. In the absence of any real log-periodic structure in the data, there is no basis for expecting the nonlinear parameters to satisfy their criteria. If we blindly apply a complicated regression specification to a data set, we will necessarily obtain parameter estimates, but these estimates will be meaningless if the specification is wrong.

SJ put great faith in the values of $\beta$ and $\omega$ that they find in their many examples of log-periodic spells, and they are quite impressed that they always 
manage to get similar values. However, we do not replicate this result with our own procedure. For example, our best fit for the 80-87 data set produces very different values of $\beta$ and $\omega$ from what Johansen et al report in [4]. One possible explanation for this discrepancy, suggested by their criticism mentioned above, is that they are constraining their fitting procedure to consider only values of the nonlinear parameters which fall into a chosen set. If this is what they are doing, it would generally be considered inappropriate and would cloud the issue of whether they are actually estimating the population parameters they believe they are estimating.

Regarding the Lomb periodograms of [3], we erroneously stated in [1] that this procedure offered an independent means of estimating the frequency of a log-periodic fit. In fact, the method described in [3] is not independent from their curve-fitting procedure since they use the fit to determine $t_{c}$ and to detrend the data. It would be very surprising indeed if they found a low power at the frequency $\omega$ after they have already shown they can fit the data to an oscillation with frequency $\omega$. Consequently, the consistency of their Lomb periodograms with their curve fitting does not help to corroborate SJ's claims.

In [5], SJ also express some outrage regarding our dismissal of previous statistical analyses carried out by Johansen et al [3- [4] and by Feigenbaum and Freund 11]. We stand by our comments from the first paper. With respect to Monte Carlo simulations, we can only use them to establish statistical properties of a stochastic process if the data-generating process (DGP) used in these simulations shares those properties. The fact that simulations produce behavior different from what we observe in real markets only proves that the DGP driving these simulations is not the true DGP.

With respect to studies involving fits of out-of-sample data, many of SJ's supposed fits are not impressive. Just leafing through the figures in [5], we find examples where the alleged log-periodic oscillations are dwarfed by the volatility in the data and it is very hard to believe we are looking at the best fit to satisfy the specification (3), which may not even be a correct specification. Financial economists can tell many stories of the folly in putting too much faith in patterns discovered by poring through out-of-sample data. If log-periodicity is truly a harbinger of crashes, this is not going to be established with data mining. Its power as a forecasting tool can only be measured by its ability to forecast. We must wait to see what the future brings to judge this issue.

That said, SJ do present one interesting new piece of evidence in [5]. They describe the result of a systematic scan of data for the Hang-Seng index using a moving window of 1.5 years from 1980 to 1999. During this period, they found 9 examples of log-periodic spells that satisfy their criterion. All but one of these was followed by a drawdown of $5 \%$ or more, a result so good that it should make one suspicious. This was also a period in which they claim at least eight (independent) drawdowns of $10 \%$ or more occurred. Clearly, the Hang-Seng is a highly volatile index. The average time between these large drawdowns is about 2.5 years, about the same order of magnitude of their window size.

Now, of course, SJ would argue that this is great evidence of the power of log-periodicity to predict crashes. But they give no information about how 
well the estimation of $t_{c}$ using their procedure does at estimating the actual time of the ensuing crash, and this suggests another mechanism might be at work here. It is possible that their selection criteria will, with great probability, rule out time periods in which a large drawdown occurs. If that is the case, their procedure may simply be flagging periods in which crashes do not occur. And since crashes are very frequent in this 20-year interval, the time between a flagged period and the next crash is likely to be short, so it may look like their procedure is forecasting crashes when it is not really doing that at all.

\section{Behavior of First Differences}

The most important section of 11, where we made our main original contribution, received the least attention from SJ in [5]. As we already noted in the previous section, if we assume $\ln p$ is following a process of the form (11) then least squares estimators of the parameters in Eq. (3) can give spurious results if one regresses $\ln p$ directly on functions of time. Instead, the appropriate procedure is to look at the first differences of $\ln p$. We did this in Section 4 of [1] for the log-periodic spell in the S\&P 500 leading up to the October 1987 crash.

As we discussed in the previous paper, the least-squares objective function for the specification (3) does not have a clearly defined global minimum with respect to the nonlinear parameters, so it is not clear how one should properly estimate the mean and, more importantly, the variance of least-squares estimators for these nonlinear parameters. We, therefore, focused on the linear parameters $A, B$, and $C$. It is well accepted in the finance literature that the noise-term $\varepsilon(t)$ in (1) is dependent on previous values of $\varepsilon$, which would invalidate standard error formulas for the estimation of $A, B$, and $C$. (SJ $[5]$ insinuate that, because the distribution of $\varepsilon$ is fat-tailed as opposed to Gaussian, least squares methods are inappropriate for estimation in this context. However, consistent estimation with least squares methods does not require Gaussian noise distributions, which are by far the exception in econometrics.) To overcome the problem of serial dependence, we estimated critical values for the $T$ statistics of the linear parameters using a Monte Carlo simulation. This procedure requires that we specify a DGP to model the behavior of the S\&P 500. We used a random walk for this purpose. While we do not believe the S\&P 500 actually follows a random walk, we can set the parameters of the random walk to match some of the properties of the $\mathrm{S} \& \mathrm{P} 500$, in this case the first two moments.

In [5], SJ make note of an apparent inconsistency here. In [1], we dismissed their Monte Carlo results because they only considered one possible DGP for securities prices, a GARCH model. Yet here, we expect them to accept our own Monte Carlo results which also consider only one DGP.

There is a subtle point of reasoning that SJ evidently missed here. In their case, they are trying to persuade readers to accept an alternative hypothesis. In contrast, we were testing and rejecting such a hypothesis. As they 
admit, "No truth is ever demonstrated in science; the only thing that can be done is to construct models and reject them at a given level of statistical significance." In classical statistics, one either rejects a hypothesis or one fails to reject it. In their case, they rejected the null hypothesis that a GARCH model could produce the behavior they observed in real markets. If their experiment had turned out the other way, we would probably not be writing this paper because people would have concluded there was nothing extraordinary about log-periodic fits. Instead, the experiment went as they hoped, and we can conclude that the actual stochastic process is not a GARCH process. That is all we can conclude. We cannot draw any conclusions about the validity of logperiodicity because the space of possible DGPs is huge, and all they ruled out is an infinitesimal fraction of it. Note also that the fact that the GARCH model is "one of the most fundamental benchmarks of the industry" is irrelevant. The GARCH model is a very good model of how stock prices behave under normal circumstances, but SJ's own investigations demonstrate how poorly it captures the behavior of stock prices under the extreme circumstances surrounding a large drawdown.

In contrast to their rejection of the GARCH, we failed to reject the null hypothesis that a random walk could produce the log-periodic behavior we were looking at. We can, therefore, conclude that the random walk-like properties of the true DGP are enough to produce this behavior. It is reasonable to expect that other DGPs with similar properties can also replicate this behavior. Whereas SJ have merely eliminated from consideration one out of an infinite number of possible DGPs, we have established that the observed log-periodic behavior in first differences is not extraordinary. Thus, the negative result of our paper speaks much louder than the positive results that they and others have found.

We tested the specification

$$
\Delta \ln p\left(t_{i}\right)=0=A+B \Delta f_{1}\left(t_{i}\right)+C \Delta f_{2}\left(t_{i}\right)+\sum_{s=2}^{4} D_{s} \delta_{s i}+\varepsilon_{i},
$$

where we had closing prices sampled at $N$ dates $t_{1}, \ldots, t_{N}$ and $\varepsilon_{i}$ is a meanzero noise term. The covariate $\delta_{s i}$ is a delta function equal to 1 if $\Delta t_{i}=s$ and 0 otherwise. Note that in the Sornette-Johansen paradigm the constant $A$ and the time dummy variables $D_{2}, D_{3}$, and $D_{4}$ will vanish. One criticism of SJ's work is that they have done no specification tests. They assume their hypothetical specification is the correct one and do not consider the possibility that an alternative might do better, which would constitute a rejection of their hypothesis. The inclusion of these extra terms here allows for the possibility that the upward trend seen in most stock prices leading up to a drawdown results from a constant drift rather than the more complicated $f_{1}$ term of their specification.

For the period from January 1980 to September 1987, we found that $B$, the coefficient of $\Delta f_{1}$, was not statistically significant. Thirty percent of our Monte Carlo simulations produced a $T$ statistic $B$ equal or larger in magnitude 
than what we obtained for our best fit. In [5], SJ dismiss this finding, attributing the insignificance of $f_{1}$ to "the intrinsic difficulty in quantifying a trend and an acceleration in very noisy data." However, one could invoke similar difficulties to dismiss the entire field of log-periodic precursors.

For $C$, the coefficient of the log-periodic term, we found that it was statistically significant at the $5 \%$ level if we considered the whole data set. However, if we restrict our attention to the period from 1980-1986, twentyfive percent of our Monte Carlo simulations produced a $C$ equal or larger in magnitude to the result of the best fit. Thus, we concluded that the logperiodic component was not statistically significant for the bulk of the logperiodic spell preceding the $87 \mathrm{crash}$. In $[5$, SJ dismiss this result also: "It is as if a worker on critical phenomena was trying to get a reliable estimation of $t_{c}$ and $\beta$ by thrashing the last $15 \%$ of the data, which are of course the most relevant." This argument presupposes that we are, in fact, looking at a critical phenomenon, which in our opinion has yet to be established. In any case, that is beside the point. If it was our purpose to measure nonlinear parameters like $t_{c}$ and $\beta$, then clearly it would be a mistake to throw out the data where the critical behavior is most pronounced. But that was not our purpose. Our purpose was to determine whether these functions had a statistically significant presence throughout the data, and the answer is they do not. Granted, this does not prove that log-periodicity is absent from the first difference series during these six years. It only proves we cannot convincingly detect log-periodicity there. Nevertheless, given this null result, a theory which can explain the logperiodic behavior in $\ln p$ without requiring $\log$-periodic behavior in $\Delta \ln p$ will be preferred over the Sornette-Johansen paradigm by Occam's Razor.

Before moving on, we must point out that SJ are being hypocritical when they chastise us for throwing out the last year of data. As we noted in [1], it is common practice in this literature to throw out from these curve-fitting procedures the end data points closest to the crash, sometimes eliminating a period as large as a few months. SJ even confess to this practice in [5]. These end data points are even closer to the alleged critical point than most of the data we excluded. If these are the crucial data points, then we have all committed a great $\sin$.

\section{The Frequency Distribution of Drawdowns}

The last subject considered in [1] was SJ's claim, first put forward in [2] and later elaborated upon in [12], that large events like stock market crashes are outliers in the frequency distribution of drawdowns. They maintained that all but the largest drawdowns fit well to an exponential or a stretched exponential distribution, and they, therefore, posited that a different mechanism must be producing these outlier events. We responded to this claim by doing a specification test on this drawdown distribution, and we found both the exponential and the stretched exponential specifications could be rejected. 
In [5], SJ answer this challenge with three new pieces of evidence. First, they describe the results of a bootstrap test. They reshuffled the sample of daily returns for the NASDAQ composite index from 1971 to 2000 and generated 10000 days of simulated data. The resulting drawdown distribution produced a much smaller frequency of large events than is observed in the real data. However, this is not surprising. They are bootstraping the unconditional distribution of daily returns, but it is well established that daily returns are dependent on previous returns, and, in fact, the stock market may even be a long memory process. All of these dependencies are ignored by this bootstrap test. We can conclude from this test that these dependencies, indeed, play an important role in producing large drawdowns, but this does not establish that large drawdowns are outliers.

Next, they describe the drawdown distribution resulting from Monte Carlo simulations of the stock market as a GARCH process. This simulated distribution also differs from the empirical distribution, but again this only substantiates their result that the true DGP underlying securities prices is not a GARCH process.

Finally, they corroborate our result that the stretched exponential does not fit well to moderate drawdowns larger than $5 \%$. But instead of abandoning the claim that large drawdowns are outliers, they expand the class of drawdowns that they consider to be outliers. To some extent, we must recognize that this is an argument over semantics. What do we mean by an outlier?

Moving beyond semantics, we do not agree with SJ's claim that "the exponential distribution is the natural null hypothesis for uncorrelated returns." In [4], Johansen et al derive the result that the drawdown distribution should be exponential under the same assumption of their bootstrap test that changes in a stock price from one instant to the next are independent. This assumption is certainly false, though it may be a reasonable approximation for small drawdowns. As the length of a chain of similarly signed innovations increases, however, the serial dependence in this chain will likely become more important. Thus, while it may be a natural null hypothesis that the distribution should be exponential in the limit of small drawdown sizes, it is not fair to extrapolate this hypothesis to large drawdown sizes. Moreover, this weaker null hypothesis that the distribution be exponential only in the small drawdown limit is supported by the preponderance of the evidence.

We also quibble with their claim that one could not "prove" that large drawdowns are outliers. As we discussed in [1], if one could reliably distinguish drawdowns with a log-periodic precursor from drawdowns lacking such a precursor, then one could determine the distribution of these two populations of drawdowns. If the distributions differ in a statistically significant manner, that would go a long way to establishing the claim that a different mechanism is responsible for the two kinds of drawdowns.

\section{Concluding Remarks}


Speaking as a researcher who has been involved from the beginning in the study of log-periodicity in the stock market, we believe that the initial evidence of crashes as critical phenomena was certainly intriguing. However, it is important not to get carried away by a new hypothesis and lose one's scientific objectivity. SJ lament [5] that "it is an all too common behavior to dismiss lightly a serious hypothesis by not taking the trouble to learn the relevant skills necessary to test it rigorously." However, they themselves have thrown roadblocks in the way of testing it rigorously. As long as they keep the details of their methodology secret, their results cannot be independently tested or reproduced. They have also disregarded many econometric procedures that would generally be considered the most appropriate tools for addressing the problems they encounter. Instead, they cobble together methods with little or no theoretical basis and then they question why nobody follows their trail. Furthermore, when one finds that standard methods produce results contrary to their hypothesis, one has to consider if maybe they have rejected these standard methods precisely because the results obtained do not support their hypothesis.

SJ also draw an analogy between log-periodicity and global warming. We think a better analogy can be made to the canalis of Mars. For over a hundred years, astronomers like Giovanni Schiaparelli and Percival Lowell were almost fanatical in their belief that they could see evidence of a great Martian civilization through their telescopes. Eventually, telescopic resolutions improved to the point where astronomers could clearly see there was no system of artificial canals crisscrossing the surface, yet it took a long time for the idea to die.

\section{Acknowledgements}

We would like to thank Simon Lee, Gene Savin, Charles Whiteman, and Robert Wickham for their input. We are also grateful to J. D. Farmer for his encouragement.

\section{References}

[1] Feigenbaum J 2001 Quant. Finance 1346

[2] Johansen A and Sornette D 1999 Eur. Phys. J. B 1141

[3] Johansen A, Sornette D and Ledoit O 1999 J. Risk 15

[4] Johansen A, Sornette D and Ledoit O 2000 Int. J. Theor. Appl. Finance 3 219

[5] Sornette D and Johansen A 2001 Preprint cond-mat/0106520 (in press at Quant. Finance)

[6] Ilinski K 1999 Int. J. Mod. Phys. C 10741 
[7] Cochrane J H 2001 Asset Pricing (Princeton, NJ: Princeton University Press)

[8] Constantinides G M 1982 J. of Business 55253.

[9] Sornette D and Ide K 2001 Preprint cond-mat/0106054

[10] Sornette D and Johansen A 1997 Physica A 245411

[11] Feigenbaum J and Freund P G O 1996 Int. J. Mod. Phys. B 103737

[12] Johansen A and Sornette D 2000 Preprint cond-mat/0010050 